\title{
Evaluation of Different Doses of Imidacloprid 60 FS - A New Seed Dressing Chemical against Sucking Pests of Greengram
}

\section{Shobharani*, Sidramappa and N.M. Sunilkumar}

Agricultural Research Station, Bidar University of Agricultural Sciences, Raichur, Karnataka, India

*Corresponding author

\section{A B S T R A C T}

\section{Keywords}

Sucking pests, Greengram, Imidacloprid.

Article Info

Accepted:

26 October 2017

Available Online:

10 December 2017
Field experiment was conducted for two consecutive seasons under rain fed condition to evaluate the efficacy of Imidacloprid 60 FS a new seed dressing chemical at different doses against greengram early season sucking pests at Agricultural Research Station, Bidar, Karnataka. Among the different doses evaluated, the higher doses of Imidacloprid 60 FS, i.e., at $10 \mathrm{ml}$ and $15 \mathrm{ml} / \mathrm{kg}$ of seeds were proved superior in protecting the crop from the early season sucking pests viz., thrips, aphids and leafhoppers up to 40-45 days after sowing and recorded significantly highest grain yield. Hence, considering the cost of plant protection, Imidacloprid 60 FS @ 10 $\mathrm{ml} / \mathrm{kg}$ of seeds can be used for managing the greengram early season sucking pests up to 40-45 days after sowing.

\section{Introduction}

Pulses are rich in proteins and are the second most important constituent of Indian diet after cereals. Among the different pulses, greengram (Vigna radiata (L.) contains $25 \%$ of protein which is one of the essential nutrients of the human diet. In addition, it is a short duration crop and suitable for dry land forming as it is drought resistant. In India, the crop is cultivated in 30.41 lakh ha with a production of 14.24 lakh tonnes. While in Karnataka it is grown in an area of about 2.53 lakh ha with production and productivity of 0.62 lakh tonnes and $247 \mathrm{~kg} / \mathrm{ha}$ respectively. A number of factors are responsible for its low yield, however, on an average, 2.5 to 3.0 million tonnes of pulses are lost annually due to pest problems (Rabindra et al., 2004). The sucking insect pests like aphids, leafhoppers, thrips and whiteflies are major pests damaging the crop since from seedling stage to pod maturing stage of the crop by sucking the sap from leaves and tender pods causing the heavy yield losses.

In order to manage these sucking pests, Farmers go for foliar application of the insecticides after colonization of the sucking pests which was not only hazardous to environment but also alters the natural enemy population. Under such situation, 
Imidacloprid, a neonicotinoid compound belonging to the chloronicotinyl insecticide which has gut and contact activity against insects (Maienfisch et. al., 2001) can be used for managing the sucking pests. Seed treatment with Imidacloprid shows systemic and residual toxicity in several crop plants and interferes with transmission of stimuli or impulses in the nervous system of insect herbivores, and gives excellent control against a broad range of commercially important sucking insect pests (Zhang et al., 2011).

Seed treatment with systemic insecticide is an integral part of pest management tactics, which is comparatively less pollutant to the environment, cost effective, selective and reported to maintain natural equilibrium (Taylor et al., 2001; Nault et al., 2004). The Imidacloprid (Gaucho $70 \mathrm{WS}$ ) is a low cost, selective and less polluting compound which is found promising against sucking pests of cotton (Patil et al., 1999; Dandale et al., 2001; Vadodaria et al., 2001; Dhawan and Simwat, 2002; Patil et al., 2004). This keeps cotton seedlings free from sucking pests at least 45 days of their age (Udikeri et al., 2007).

Seed treatment is one of the highly progressive and demandable technologies in integrated pest management (IPM) for controlling various crop pests (Taylor et al., 2001; Magalhaes et al., 2009). Therefore, the present study was undertaken to know the comparative effect of Imidacloprid (Gaucho 60 FS) as seed treating agent and other foliar insecticide on the abundance of sucking pests and yield of greengram under natural field conditions.

\section{Materials and Methods}

A field experiment was conducted to test the efficacy of different doses Imidacloprid 60 FS against sucking pests viz., aphids, thrips and leafhoppers infesting greengram. The experiment was carried out at Agricultural Research Station, Bidar during Kharif season (2014 and 2015) in medium black soil under rainfed conditions. The experiment was laid out in Randomized block design with eight treatments replicated thrice. The plot size of each treatment was $3 \times 4 \mathrm{~m}^{2}$ with $45 \mathrm{~cm} \mathrm{X} 10$ $\mathrm{cm}$ spacing. The seeds of the greengram var.BGS-9 were taken in a polythene bag and required quantity of the Imidacloprid 60 FS (@ 5, 10 and $15 \mathrm{ml} / \mathrm{kg}$ of seeds) was added to this and stirred carefully. If necessary few $\mathrm{ml}$ of water can be added to get uniform coating of the chemical on the seeds and dried in shade before sowing. Before sowing the treated seeds, there should be sufficient moisture in the soil. The insecticides, Imidacloprid $17.8 \mathrm{SL} 0.3 \mathrm{ml} / \mathrm{Lt}$, Dimethoate 30 EC1.7 ml/Lt, Neem oil (1500 ppm) @ $5 \mathrm{ml} / \mathrm{Lt}$ and NSKE $5 \%$ were used as checks for comparison. An untreated check was also maintained. All the recommended package of practices was followed to raise the crop, except plants protection measures. Where ever necessary a spray was given to control leaf eating caterpillar and pod borers.

Observations on incidence of sucking pests viz., aphids, thrips and leafhoppers were made at 10, 20, 30 and 40 days after complete emergence of seedlings in all treatments on randomly selected 5 plants/plot. The data was subjected for square root transformation and statistical analysis. The seed yield was recorded plot wise at the time of harvest and converted to hectare basis and subjected for statistical analysis. The mean comparisons were made by Duncan's multiple range test (DMRT). Visual observations were made for phytotoxic effects if any, expressed after germination.

\section{Results and Discussion}

Imidacloprid 60 FS rendered protection against early season sucking pests (Table 1). 
In general, the incidence of sucking pests was high during 2015-16 compared to 2014-15. The performance of seed treatment / dressers used in the present study was consistent in both the seasons.

The effectiveness of different doses of Imidacloprid 60 FS for seed treatments and foliar spray of different insecticides and plant products against management of sucking pests of greengram under field condition during 2014-15 is presented in the table 1. The Imidacloprid seed treatment showed significantly lower abundance of aphid population which ranged from 0.33 to 2.67 aphids per top three leaves than other foliar spray treatments which showed 10.67 to 11.33 aphids per top three leaves at 15 days after sowing. At 30 days after sowing lowest aphid population of 1.56 and 2.11aphids / top three leaves was recorded in the plots treated with Imidacloprid 60 FS @ $15 \mathrm{ml} / \mathrm{Kg}$ of seeds and $10 \mathrm{ml} / \mathrm{kg}$ of seeds respectively. These two treatments were followed by Imidacloprid 17.8 SL@0.3 ml/ Lt of water with 2.89 aphids/top three leaves, Dimethoate 30 EC @ $1.7 \mathrm{ml} / / \mathrm{Lt}$ of water with 4.28 aphids/top three leaves, Neem oil (1500 ppm) @ $5 \mathrm{ml} / \mathrm{Lt}$ of water with 4.67 aphids/top three leaves, seed treatment with Imidacloprid 60 FS @ $5 \mathrm{ml} / \mathrm{Kg}$ of seeds with 5.22 aphids/top three leaves and NSKE 5\% with 8.78 aphids/top three leaves. The highest aphid population was noticed in untreated control.

At 45 days after sowing aphid population started to build up in all the treatment which ranged from 9.11 to 14.11 aphids/top three leaves.

However, highest aphid population was recorded in untreated control with 21.89 aphids/ top three leaves. The same trend was followed at 60 days after sowing with decreasing in aphid population in all treatments.
During 2015-16 (Table 2) same trend was followed with respect the aphid population observed on top three leaves per plant in each treatment.

The thrips population at 15 days after sowing was found significantly lower in the plot (Table 1) where, seed treatment with Imidacloprid 60 FS @ 15 ml/ Kg of seeds and $10 \mathrm{ml} / \mathrm{kg}$ of seeds was done by recording 0.11 and 0.33 thrips/top three leaves respectively. This was followed by Imidacloprid60 FS @ 5 $\mathrm{ml} / \mathrm{Kg}$ of seeds with 1.56 thrips/top three leaves. Whereas the untreated plots the thrips population ranged from 6.44 to 7.00 thrips/ top three leaves. Thirty days after sowing lowest thrips population of 1.56 and 2.89 thrips / top three leaves was recorded in the plots treated with Imidacloprid 60 FS @ 15 $\mathrm{ml} / \mathrm{Kg}$ of seeds and $10 \mathrm{ml} / \mathrm{kg}$ of seeds respectively. These two treatments were followed by Imidacloprid 17.8 SL @ $0.3 \mathrm{ml} /$ Lt of water with 4.22 thrips/top three leaves, Imidacloprid 60 FS @ 5 ml/ Kg of seeds with 4.78 thrips/top three leaves, Dimethoate 30 EC @ $1.7 \mathrm{ml} / /$ Lt of water with 4.89 thrips/top three leaves, Neem oil (1500 ppm) @ 5ml/Lt of water with 5.56 thrips/top three leaves and NSKE 5\% with 6.11thrips/top three leaves. The highest thrips population was noticed in untreated control.

At 45 days after sowing thrips population started to build up in all the treatment which ranged from 5.22 to 14.89 thrips/top three leaves. However, highest thrips population was recorded in untreated control with 14.89 thrips/ top three leaves. The same trend was followed at 60 days after sowing with decreasing in thrips population in all treatments.

During 2015-16 (Table 2) same trend was followed with respect the thrips population observed on top three leaves per plant in each treatment. 
Table.1 Management of sucking pests of Greengram during 2014-15 Kharif

\begin{tabular}{|c|c|c|c|c|c|c|c|c|c|c|c|c|c|c|}
\hline \multirow[b]{2}{*}{ Treatments } & \multirow[b]{2}{*}{ Dose } & \multicolumn{4}{|c|}{$\begin{array}{l}\text { No. of aphids/ top three } \\
\text { leaves }\end{array}$} & \multicolumn{4}{|c|}{ No. of thrips/ top three leaves } & \multicolumn{4}{|c|}{$\begin{array}{l}\text { No. of leafhoppers/ top three } \\
\text { leaves }\end{array}$} & \multirow[t]{2}{*}{$\begin{array}{l}\text { Yield } \\
\text { (Qtl. } \\
\text { /ha) }\end{array}$} \\
\hline & & $\begin{array}{l}15 \\
\text { DAS }\end{array}$ & $\begin{array}{l}30 \\
\text { DAS } \\
\end{array}$ & $\begin{array}{l}45 \\
\text { DAS } \\
\end{array}$ & $\begin{array}{l}60 \\
\text { DAS } \\
\end{array}$ & $\begin{array}{l}15 \\
\text { DAS }\end{array}$ & $\begin{array}{l}30 \\
\text { DAS }\end{array}$ & $\begin{array}{l}45 \\
\text { DAS }\end{array}$ & $\begin{array}{l}60 \\
\text { DAS } \\
\end{array}$ & $\begin{array}{l}15 \\
\text { DAS }\end{array}$ & $\begin{array}{l}30 \\
\text { DAS }\end{array}$ & $\begin{array}{l}45 \\
\text { DAS }\end{array}$ & \begin{tabular}{|l}
60 \\
DAS \\
\end{tabular} & \\
\hline 1. Imidacloprid $60 \mathrm{FS}$ & $\begin{array}{l}5 \mathrm{ml} \mathrm{/} \mathrm{Kg} \\
\text { of seeds }\end{array}$ & $\begin{array}{l}2.67 \\
(1.90) \\
\end{array}$ & \begin{tabular}{|l|}
5.22 \\
$(2.49)$ \\
\end{tabular} & $\begin{array}{l}12.67 \\
(3.70) \\
\end{array}$ & $\begin{array}{l}6.56 \\
(2.73) \\
\end{array}$ & $\begin{array}{l}1.56 \\
(1.58) \\
\end{array}$ & $\begin{array}{l}4.78 \\
(2.40) \\
\end{array}$ & $\begin{array}{l}8.78 \\
(3.12) \\
\end{array}$ & $\begin{array}{l}4.22 \\
(2.28) \\
\end{array}$ & \begin{tabular}{|l|l}
1.33 \\
$(1.53)$ \\
\end{tabular} & \begin{tabular}{|l|}
2.56 \\
$(1.87)$ \\
\end{tabular} & $\begin{array}{l}4.56 \\
(2.35) \\
\end{array}$ & $\begin{array}{l}2.22 \\
(1.78)\end{array}$ & $7.57 \mathrm{ef}$ \\
\hline 2. Imidacloprid $60 \mathrm{FS}$ & $\begin{array}{l}10 \mathrm{ml} / \mathrm{Kg} \\
\text { of seeds }\end{array}$ & \begin{tabular}{|l|}
0.89 \\
$(1.37)$ \\
\end{tabular} & \begin{tabular}{|l|}
2.11 \\
$(1.74)$ \\
\end{tabular} & \begin{tabular}{|l|}
9.44 \\
$(3.22)$ \\
\end{tabular} & $\begin{array}{l}5.67 \\
(2.58) \\
\end{array}$ & \begin{tabular}{|l|}
0.33 \\
$(1.15)$ \\
\end{tabular} & \begin{tabular}{|l|}
2.89 \\
$(1.96)$ \\
\end{tabular} & \begin{tabular}{|l|}
6.56 \\
$(2.75)$ \\
\end{tabular} & \begin{tabular}{|l|}
3.33 \\
$(2.06)$ \\
\end{tabular} & \begin{tabular}{|l|}
0.78 \\
$(1.33)$ \\
\end{tabular} & \begin{tabular}{|l|}
1.78 \\
$(1.67)$ \\
\end{tabular} & $\begin{array}{l}2.89 \\
(1.97) \\
\end{array}$ & $\begin{array}{l}1.89 \\
(1.70) \\
\end{array}$ & $12.66 \mathrm{ab}$ \\
\hline 3. Imidacloprid $60 \mathrm{FS}$ & $\begin{array}{l}15 \mathrm{ml} / \mathrm{Kg} \\
\text { of seeds }\end{array}$ & \begin{tabular}{|l|}
0.33 \\
$(1.15)$ \\
\end{tabular} & \begin{tabular}{|l|}
1.56 \\
$(1.60)$
\end{tabular} & \begin{tabular}{|l}
9.11 \\
$(3.16)$ \\
\end{tabular} & $\begin{array}{l}5.00 \\
(2.44) \\
\end{array}$ & $\begin{array}{l}0.11 \\
(1.05) \\
\end{array}$ & $\begin{array}{l}1.56 \\
(1.58) \\
\end{array}$ & \begin{tabular}{|l|}
5.22 \\
$(2.47)$ \\
\end{tabular} & \begin{tabular}{|l}
2.11 \\
$(1.76)$ \\
\end{tabular} & \begin{tabular}{|l|}
0.56 \\
$(1.25)$ \\
\end{tabular} & $\begin{array}{l}1.11 \\
(1.45) \\
\end{array}$ & $\begin{array}{l}2.56 \\
(1.89) \\
\end{array}$ & $\begin{array}{l}1.67 \\
(1.63) \\
\end{array}$ & $14.29 \mathrm{a}$ \\
\hline $\begin{array}{l}\text { 4. Imidacloprid } 17.8 \\
\text { SL }\end{array}$ & $0.3 \mathrm{ml} / \mathrm{Lt}$ & \begin{tabular}{|l}
11.11 \\
$(3.46)$ \\
\end{tabular} & $\begin{array}{l}2.89 \\
(1.96) \\
\end{array}$ & \begin{tabular}{|l|}
9.89 \\
$(3.30)$ \\
\end{tabular} & $\begin{array}{l}5.33 \\
(2.49) \\
\end{array}$ & $\begin{array}{l}6.89 \\
(2.79) \\
\end{array}$ & \begin{tabular}{|l}
4.22 \\
$(2.28)$ \\
\end{tabular} & \begin{tabular}{|l|}
6.89 \\
$(2.81)$ \\
\end{tabular} & \begin{tabular}{|l}
3.44 \\
$(2.11)$ \\
\end{tabular} & $\begin{array}{l}4.56 \\
(2.36) \\
\end{array}$ & $\begin{array}{l}2.44 \\
(1.85) \\
\end{array}$ & $\begin{array}{l}3.00 \\
(1.99) \\
\end{array}$ & $\begin{array}{l}1.67 \\
(1.63) \\
\end{array}$ & $10.77 \mathrm{c}$ \\
\hline $\begin{array}{l}\text { 5. Neem oil (1500 } \\
\text { ppm) }\end{array}$ & $5 \mathrm{ml} / \mathrm{Lt}$ & $\begin{array}{l}10.67 \\
(3.41) \\
\end{array}$ & \begin{tabular}{|l}
4.67 \\
$(2.37)$ \\
\end{tabular} & \begin{tabular}{|l}
12.78 \\
$(3.71)$ \\
\end{tabular} & $\begin{array}{l}6.22 \\
(2.68) \\
\end{array}$ & \begin{tabular}{|l}
6.44 \\
$(2.72)$ \\
\end{tabular} & \begin{tabular}{|l}
5.56 \\
$(2.56)$ \\
\end{tabular} & \begin{tabular}{|l}
8.67 \\
$(3.11)$ \\
\end{tabular} & \begin{tabular}{|l}
4.89 \\
$(2.42)$ \\
\end{tabular} & \begin{tabular}{|l|}
4.33 \\
$(2.30)$ \\
\end{tabular} & \begin{tabular}{|l|}
2.89 \\
$(1.96)$ \\
\end{tabular} & $\begin{array}{l}4.67 \\
(2.38) \\
\end{array}$ & $\begin{array}{l}2.11 \\
(1.76) \\
\end{array}$ & $9.30 \mathrm{de}$ \\
\hline 6. NSKE & $5 \%$ & $\begin{array}{l}10.78 \\
(3.43) \\
\end{array}$ & \begin{tabular}{|l}
8.78 \\
$(3.12)$ \\
\end{tabular} & \begin{tabular}{|l|}
14.11 \\
$(3.87)$ \\
\end{tabular} & $\begin{array}{l}7.11 \\
(2.84) \\
\end{array}$ & \begin{tabular}{|l|}
6.89 \\
$(2.81)$ \\
\end{tabular} & \begin{tabular}{|l|}
6.11 \\
$(2.66)$ \\
\end{tabular} & $\begin{array}{l}10.22 \\
(3.34) \\
\end{array}$ & \begin{tabular}{|l|}
5.33 \\
$(2.51)$ \\
\end{tabular} & \begin{tabular}{|l|}
4.78 \\
$(2.40)$ \\
\end{tabular} & $\begin{array}{l}4.00 \\
(2.24) \\
\end{array}$ & $\begin{array}{l}5.67 \\
(2.58) \\
\end{array}$ & $\begin{array}{l}2.89 \\
(1.97) \\
\end{array}$ & $6.13 \mathrm{fg}$ \\
\hline 7. Dimethoate $30 \mathrm{EC}$ & $1.7 \mathrm{ml} / \mathrm{Lt}$ & $\begin{array}{l}11.33 \\
(3.51) \\
\end{array}$ & \begin{tabular}{|l|}
4.28 \\
$(2.29)$ \\
\end{tabular} & \begin{tabular}{|l|}
11.11 \\
$(3.47)$ \\
\end{tabular} & $\begin{array}{l}5.78 \\
(2.60) \\
\end{array}$ & \begin{tabular}{|l|}
7.00 \\
$(2.82)$ \\
\end{tabular} & \begin{tabular}{|l|}
4.89 \\
$(2.42)$ \\
\end{tabular} & \begin{tabular}{|l|}
7.33 \\
$(2.88)$ \\
\end{tabular} & \begin{tabular}{|l|}
4.89 \\
$(2.41)$ \\
\end{tabular} & \begin{tabular}{|l|}
4.11 \\
$(2.26)$ \\
\end{tabular} & $\begin{array}{l}2.67 \\
(1.90) \\
\end{array}$ & $\begin{array}{l}3.89 \\
(2.20) \\
\end{array}$ & $\begin{array}{l}1.9 \\
(1.70) \\
\end{array}$ & $9.90 \mathrm{~cd}$ \\
\hline \multirow[t]{4}{*}{ 8. Control } & - & \begin{tabular}{|l}
10.69 \\
$(3.42)$ \\
\end{tabular} & \begin{tabular}{|l|}
15.22 \\
$(4.02)$ \\
\end{tabular} & \begin{tabular}{|l|}
21.89 \\
$(4.78)$ \\
\end{tabular} & $\begin{array}{l}16.56 \\
(4.19) \\
\end{array}$ & \begin{tabular}{|l|}
6.67 \\
$(2.77)$ \\
\end{tabular} & $\begin{array}{l}10.11 \\
(3.33) \\
\end{array}$ & \begin{tabular}{|l|}
14.89 \\
$(3.98)$ \\
\end{tabular} & \begin{tabular}{|l|}
9.78 \\
$(3.28)$ \\
\end{tabular} & \begin{tabular}{|l|}
4.00 \\
$(2.23)$ \\
\end{tabular} & \begin{tabular}{|l|}
7.78 \\
$(2.96)$ \\
\end{tabular} & \begin{tabular}{|l|}
9.78 \\
$(3.28)$ \\
\end{tabular} & \begin{tabular}{|l}
5.00 \\
$(2.44)$ \\
\end{tabular} & $4.68 \mathrm{gh}$ \\
\hline & SEm \pm & 0.14 & 0.11 & 0.17 & 0.16 & 0.12 & 0.12 & 0.14 & 0.12 & 0.08 & 0.09 & 0.10 & 0.08 & 0.6 \\
\hline & $\mathrm{CD}(\mathbf{0 . 0 5})$ & 0.43 & 0.35 & 0.51 & 0.48 & 0.37 & 0.37 & 0.43 & 0.35 & 0.24 & 0.26 & 0.31 & 0.24 & 1.82 \\
\hline & $\mathrm{CV}(\%)$ & 9.02 & 8.13 & 8.01 & 9.72 & 9.57 & 8.79 & 7.96 & 8.59 & 7.09 & 7.60 & 7.59 & 7.50 & 11.33 \\
\hline
\end{tabular}

DAS - Days After Sowing, Figures in the parentheses are square root transformed values 
Table.2 Management of sucking pests of Greengram during 2015-16 Kharif

\begin{tabular}{|c|c|c|c|c|c|c|c|c|c|c|c|c|c|c|}
\hline \multirow[b]{2}{*}{ Treatments } & \multirow[b]{2}{*}{ Dose } & \multicolumn{4}{|c|}{ No. of aphids/ top three leaves } & \multicolumn{4}{|c|}{ No. of thrips/ top three leaves } & \multicolumn{4}{|c|}{$\begin{array}{l}\text { No. of leafhoppers/ top three } \\
\text { leaves }\end{array}$} & \multirow[t]{2}{*}{$\begin{array}{l}\text { Yield } \\
\text { (Qtl. } \\
\text { /ha) }\end{array}$} \\
\hline & & $\begin{array}{l}15 \\
\text { DAS }\end{array}$ & \begin{tabular}{|l}
30 \\
DAS \\
\end{tabular} & $\begin{array}{l}45 \\
\text { DAS }\end{array}$ & $\begin{array}{l}60 \\
\text { DAS }\end{array}$ & $\begin{array}{l}15 \\
\text { DAS }\end{array}$ & $\begin{array}{l}30 \\
\text { DAS }\end{array}$ & $\begin{array}{l}45 \\
\text { DAS }\end{array}$ & \begin{tabular}{|l}
60 \\
DAS \\
\end{tabular} & $\begin{array}{l}15 \\
\text { DAS }\end{array}$ & $\begin{array}{l}30 \\
\text { DAS }\end{array}$ & $\begin{array}{l}45 \\
\text { DAS }\end{array}$ & $\begin{array}{l}60 \\
\text { DAS }\end{array}$ & \\
\hline $\begin{array}{l}\text { 1. Imidacloprid } 60 \\
\text { FS }\end{array}$ & $\begin{array}{l}5 \mathrm{ml} / \mathrm{Kg} \\
\text { of seeds }\end{array}$ & \begin{tabular}{|l}
2.11 \\
$(1.76)$ \\
\end{tabular} & $\begin{array}{l}5.67 \\
(2.58) \\
\end{array}$ & $\begin{array}{l}10.33 \\
(3.36)\end{array}$ & $\begin{array}{l}8.78 \\
(3.12) \\
\end{array}$ & $\begin{array}{l}1.11 \\
(1.45) \\
\end{array}$ & $\begin{array}{l}3.26 \\
(2.05) \\
\end{array}$ & $\begin{array}{l}7.67 \\
(2.94) \\
\end{array}$ & \begin{tabular}{|l}
5.78 \\
$(2.60)$ \\
\end{tabular} & $\begin{array}{l}2.22 \\
(1.79) \\
\end{array}$ & $\begin{array}{l}4.22 \\
(2.28) \\
\end{array}$ & $\begin{array}{l}5.11 \\
(2.47) \\
\end{array}$ & $\begin{array}{l}2.67 \\
(1.90) \\
\end{array}$ & $7.93 \mathrm{f}$ \\
\hline $\begin{array}{l}\text { 2. Imidacloprid } 60 \\
\text { FS }\end{array}$ & $\begin{array}{l}10 \mathrm{ml} / \mathrm{Kg} \\
\text { of seeds }\end{array}$ & $\begin{array}{l}1.00 \\
(1.41) \\
\end{array}$ & $\begin{array}{l}2.44 \\
(1.84) \\
\end{array}$ & $\begin{array}{l}8.11 \\
(3.02) \\
\end{array}$ & $\begin{array}{l}6.00 \\
(2.64) \\
\end{array}$ & $\begin{array}{l}0.56 \\
(1.25) \\
\end{array}$ & $\begin{array}{l}1.44 \\
(1.56) \\
\end{array}$ & $\begin{array}{l}4.89 \\
(2.42) \\
\end{array}$ & $\begin{array}{l}3.00 \\
(2.00) \\
\end{array}$ & $\begin{array}{l}0.89 \\
(1.37) \\
\end{array}$ & $\begin{array}{l}2.00 \\
(1.72)\end{array}$ & $\begin{array}{l}3.11 \\
(2.02)\end{array}$ & $\begin{array}{l}1.78 \\
(1.67) \\
\end{array}$ & $13.56 \mathrm{ab}$ \\
\hline $\begin{array}{l}\text { 3. Imidacloprid } 60 \\
\text { FS }\end{array}$ & $\begin{array}{l}15 \mathrm{ml} / \mathrm{Kg} \\
\text { of seeds }\end{array}$ & \begin{tabular}{|l|l}
0.56 \\
$(1.25)$ \\
\end{tabular} & $\begin{array}{l}1.89 \\
(1.70)\end{array}$ & $\begin{array}{l}7.78 \\
(2.96) \\
\end{array}$ & $\begin{array}{l}5.89 \\
(2.62) \\
\end{array}$ & $\begin{array}{l}0.22 \\
(1.10)\end{array}$ & $\begin{array}{l}1.11 \\
(1.45) \\
\end{array}$ & $\begin{array}{l}4.44 \\
(2.33) \\
\end{array}$ & \begin{tabular}{|l|}
2.78 \\
$(1.94)$ \\
\end{tabular} & \begin{tabular}{|l|}
0.78 \\
$(1.33)$
\end{tabular} & $\begin{array}{l}1.56 \\
(1.58) \\
\end{array}$ & \begin{tabular}{|l|}
3.11 \\
$(2.02)$ \\
\end{tabular} & $\begin{array}{ll}0.89 \\
(1.37)\end{array}$ & $14.96 \mathrm{a}$ \\
\hline $\begin{array}{l}\text { 4. Imidacloprid } 17.8 \\
\text { SL }\end{array}$ & $\begin{array}{lll}0.3 & \mathrm{ml} & \\
\mathrm{Lt} & & \\
\end{array}$ & $\begin{array}{l}13.44 \\
(3.80) \\
\end{array}$ & $\begin{array}{l}3.89 \\
(2.20)\end{array}$ & $\begin{array}{l}10.00 \\
(3.30)\end{array}$ & $\begin{array}{l}7.22 \\
(2.86) \\
\end{array}$ & $\begin{array}{l}7.44 \\
(2.90)\end{array}$ & $\begin{array}{l}3.22 \\
(2.05) \\
\end{array}$ & $\begin{array}{l}6.11 \\
(2.67) \\
\end{array}$ & \begin{tabular}{|l|}
3.33 \\
$(3.33)$ \\
\end{tabular} & \begin{tabular}{|l|}
5.00 \\
$(2.45)$ \\
\end{tabular} & $\begin{array}{l}2.56 \\
(1.89) \\
\end{array}$ & \begin{tabular}{|l|}
3.89 \\
$(2.21)$ \\
\end{tabular} & \begin{tabular}{|l}
0.67 \\
$(1.29)$
\end{tabular} & $12.16 \mathrm{bc}$ \\
\hline $\begin{array}{l}\text { 5. Neem oil (1500 } \\
\text { ppm) }\end{array}$ & $5 \mathrm{ml} / \mathrm{Lt}$ & \begin{tabular}{|l|}
13.67 \\
$(3.83)$ \\
\end{tabular} & $\begin{array}{l}6.00 \\
(2.64) \\
\end{array}$ & $\begin{array}{l}12.56 \\
(3.68) \\
\end{array}$ & $\begin{array}{l}8.33 \\
(3.05) \\
\end{array}$ & $\begin{array}{l}7.56 \\
(2.92) \\
\end{array}$ & $\begin{array}{l}4.56 \\
(2.35) \\
\end{array}$ & $\begin{array}{l}8.33 \\
(3.05) \\
\end{array}$ & $\begin{array}{l}5.11 \\
(2.47) \\
\end{array}$ & $\begin{array}{l}5.22 \\
(2.48) \\
\end{array}$ & $\begin{array}{l}3.44 \\
(2.11) \\
\end{array}$ & \begin{tabular}{|l|}
4.78 \\
$(2.40)$ \\
\end{tabular} & $\begin{array}{l}1.89 \\
(1.70) \\
\end{array}$ & $10.05 \mathrm{de}$ \\
\hline 6. NSKE & $5 \%$ & $\begin{array}{l}12.78 \\
(3.71) \\
\end{array}$ & $\begin{array}{l}8.56 \\
(3.09) \\
\end{array}$ & $\begin{array}{l}15.33 \\
(4.04) \\
\end{array}$ & $\begin{array}{l}10.11 \\
(3.33) \\
\end{array}$ & $\begin{array}{l}8.00 \\
(3.00) \\
\end{array}$ & $\begin{array}{l}6.67 \\
(2.77) \\
\end{array}$ & $\begin{array}{l}9.56 \\
(3.25) \\
\end{array}$ & \begin{tabular}{|l|}
4.56 \\
$(2.35)$ \\
\end{tabular} & $\begin{array}{l}5.44 \\
(2.53) \\
\end{array}$ & $\begin{array}{l}4.56 \\
(2.36) \\
\end{array}$ & \begin{tabular}{|l}
5.56 \\
$(2.56)$ \\
\end{tabular} & $\begin{array}{l}2.33 \\
(1.82) \\
\end{array}$ & $6.89 \mathrm{fg}$ \\
\hline 7. Dimethoate $30 \mathrm{EC}$ & $\begin{array}{lll}1.7 & \mathrm{ml} \\
\mathrm{Lt} & & \\
\end{array}$ & $\begin{array}{l}13.33 \\
(3.78) \\
\end{array}$ & $\begin{array}{l}5.11 \\
(2.47) \\
\end{array}$ & $\begin{array}{l}11.56 \\
(3.54) \\
\end{array}$ & $\begin{array}{l}8.56 \\
(3.09) \\
\end{array}$ & $\begin{array}{l}7.78 \\
(2.96) \\
\end{array}$ & $\begin{array}{l}4.89 \\
(2.42) \\
\end{array}$ & $\begin{array}{l}6.89 \\
(2.81) \\
\end{array}$ & $\begin{array}{l}4.11 \\
(2.26) \\
\end{array}$ & $\begin{array}{l}4.89 \\
(2.42) \\
\end{array}$ & $\begin{array}{l}3.11 \\
(2.03) \\
\end{array}$ & $\begin{array}{l}4.00 \\
(2.23) \\
\end{array}$ & $\begin{array}{l}1.33 \\
(1.53) \\
\end{array}$ & $11.11 \mathrm{~cd}$ \\
\hline \multirow[t]{4}{*}{ 8. Control } & - & \begin{tabular}{|l|}
13.44 \\
$(3.79)$ \\
\end{tabular} & $\begin{array}{l}21.22 \\
(4.71) \\
\end{array}$ & $\begin{array}{l}28.11 \\
(5.39) \\
\end{array}$ & $\begin{array}{l}18.78 \\
(4.44) \\
\end{array}$ & $\begin{array}{l}7.00 \\
(2.81) \\
\end{array}$ & $\begin{array}{l}10.67 \\
(3.41) \\
\end{array}$ & $\begin{array}{l}14.56 \\
(3.94) \\
\end{array}$ & \begin{tabular}{|l|}
9.89 \\
$(3.30)$ \\
\end{tabular} & $\begin{array}{l}5.22 \\
(2.49) \\
\end{array}$ & $\begin{array}{l}8.33 \\
(3.05) \\
\end{array}$ & $\begin{array}{l}12.22 \\
(3.64) \\
\end{array}$ & \begin{tabular}{|l}
4.78 \\
$(2.40)$ \\
\end{tabular} & $5.13 \mathrm{gh}$ \\
\hline & SEm \pm & 0.11 & 0.10 & 0.15 & 0.12 & 0.10 & 0.09 & 0.10 & 0.08 & 0.09 & 0.10 & 0.10 & 0.07 & 0.53 \\
\hline & $\mathrm{CD}(0.05)$ & 0.33 & 0.31 & 0.46 & 0.37 & 0.31 & 0.29 & 0.29 & 0.25 & 0.27 & 0.32 & 0.31 & 0.22 & 1.62 \\
\hline & $\mathrm{CV}(\%)$ & 6.46 & 6.72 & 7.13 & 6.77 & 7.69 & 7.21 & 5.65 & 6.13 & 7.23 & 8.50 & 7.18 & 7.42 & 9.27 \\
\hline
\end{tabular}

DAS - Days After Sowing, Figures in the parentheses are square root transformed values 
Table.3 Cost economics of different seed treatment chemicals and plant products tested against sucking pests on Greengram

\begin{tabular}{|c|c|c|c|c|c|c|c|c|}
\hline Treatments & Dose & $\begin{array}{l}\text { Yield } \\
\text { (q/ha) }\end{array}$ & $\begin{array}{l}\text { Common cost } \\
\text { of cultivation }\end{array}$ & $\begin{array}{l}\text { Treatment } \\
\text { cost }\end{array}$ & $\begin{array}{l}\text { Total } \\
\text { cost }\end{array}$ & $\begin{array}{l}\text { Gross } \\
\text { returns }\end{array}$ & $\begin{array}{l}\text { Treatment } \\
\text { benefit }\end{array}$ & $\begin{array}{l}\text { B:C } \\
\text { ratio }\end{array}$ \\
\hline 1. Imidacloprid 60 FS & $\begin{array}{l}5 \mathrm{ml} / \mathrm{Kg} \text { of } \\
\text { seeds }\end{array}$ & $7.75 \mathrm{f}$ & 19776 & 320 & 20096 & 54250 & 19880.00 & 2.70 \\
\hline 2. Imidacloprid $60 \mathrm{FS}$ & $\begin{array}{l}10 \mathrm{ml} / \mathrm{Kg} \\
\text { of seeds }\end{array}$ & $13.11 \mathrm{ab}$ & 19776 & 620 & 20396 & 91770 & 57400.00 & 4.50 \\
\hline 3. Imidacloprid $60 \mathrm{FS}$ & $\begin{array}{l}15 \mathrm{ml} / \mathrm{Kg} \text { of } \\
\text { seeds }\end{array}$ & $14.63 \mathrm{a}$ & 19776 & 920 & 20696 & 102375 & 68005.00 & 4.95 \\
\hline 4. Imidacloprid 17.8 SL & $0.3 \mathrm{ml} / \mathrm{lt}$ & $11.47 \mathrm{bc}$ & 19776 & 1000 & 20776 & 80290 & 45920.00 & 3.86 \\
\hline 5. Neem oil (1500 ppm) & $5 \mathrm{ml} / \mathrm{lt}$ & $9.68 \mathrm{de}$ & 19776 & 1400 & 21176 & 67760 & 33390.00 & 3.20 \\
\hline 6. NSKE $5 \%$ & $5 \%$ & $6.51 \mathrm{fg}$ & 19776 & 875 & 20651 & 45570 & 11200.00 & 2.21 \\
\hline 7. Dimethoate 30 EC & $1.7 \mathrm{ml} / \mathrm{lt}$ & $10.51 \mathrm{~cd}$ & 19776 & 783 & 20559 & 73570 & 39200.00 & 3.58 \\
\hline 8. Control & - & $4.91 \mathrm{gh}$ & 19776 & 0 & 19776 & 34370 & 0.00 & 1.74 \\
\hline
\end{tabular}

Imidacloprid 60 FS - Rs. 4000/Lt, Imidacloprid 17.8 SL - Rs. 2840/Lt, Neem oil - Rs. 400 / Lt, Neem seed kernel - Rs. 15/Kg

Dimethoate 30 EC - Rs. 375/Lt, Greengram - Rs 7000/ Qt1., Labour cost - Rs 341.93/Lb/Day 
The effectiveness of seed dressing/ treatment chemicals against leafhopper was more or less similar to that of the aphids and thrips. Seed treatment with Imidacloprid 60 FS showed lowest leafhopper population which ranged from 0.56 to 1.33 Leafhopper/ top three leaves than other foliar spray treatments which showed 4.33 to 4.78 Leafhopper/ top three leaves at 15 days after sowing.

At 30 days after sowing lowest Leafhopper population of 1.11 and 1.78 Leafhopper / top three leaves was recorded in the plots treated with Imidacloprid 60 FS @ $15 \mathrm{ml} / \mathrm{Kg}$ of seeds and $10 \mathrm{ml} / \mathrm{kg}$ of seeds respectively.

These two treatments were followed by Imidacloprid 60 FS @ $5 \mathrm{ml} / \mathrm{Kg}$ of seeds with 2.56 Leafhopper /top three leaves, Imidacloprid 17.8 SL@0.3 ml/ Lt of water with 2.44 Leafhopper/top three leaves, Dimethoate 30 EC @ $1.7 \mathrm{ml} / /$ Lt of water with2.67 Leafhopper/top three leaves, Neem oil (1500 ppm) @ 5ml/Lt of water with 2.89 Leafhopper/top three leaves and NSKE 5\% with 4.00 Leafhopper /top three leaves. The highest leafhopper population of 7.78 Leafhopper /top three leaves was noticed in untreated control.

At 45 days after sowing Leafhopper population started to build up in all the treatment which ranged from 2.56 to 9.78 Leafhopper/top three leaves. However, highest Leafhopper population was recorded in untreated control with 9.78 Leafhopper/ top three leaves.

The same trend was followed at 60 days after sowing with decreasing in Leafhopper population in all treatments.

During 2015-16 (Table 2) same trend was followed with respect the Leafhopper population observed on top three leaves per plant in each treatment.

\section{Yield}

The highest average grain yield of 2014-15 and 2015-16 was recorded in Imidacloprid 60 FS@ $15 \mathrm{ml} / \mathrm{Kg}$ of seeds (14.63 q/ha) and Imidacloprid $60 \mathrm{FS} @ 10 \mathrm{ml} / \mathrm{kg}$ of seeds (13.11 q/ha) (Table 3). The treatment Imidacloprid 17.8 SL @ $0.3 \mathrm{ml} / \mathrm{Lt}$ of water, Dimethoate 30 EC @ $1.7 \mathrm{ml} / / \mathrm{Lt}$ of water, Neem oil (1500 ppm) @ 5ml/Lt of water, seed treatment with Imidacloprid 60 FS @ 5 $\mathrm{ml} / \mathrm{Kg}$ of seeds and NSKE 5\% recorded $11.47 \mathrm{q} / \mathrm{ha}, \quad 10.51 \mathrm{q} / \mathrm{ha}, \quad 9.68 \mathrm{q} / \mathrm{ha}, 7.75$ q/ha,6.51 q/ha,4.91 q/ha respectively. The highest aphid population was noticed in untreated control. Untreated control recorded lowest grain yield of $4.91 \mathrm{q} / \mathrm{ha}$.

\section{B: C ratio}

Imidacloprid 60 FS @ 15 ml/ Kg of seeds and Imidacloprid 60 FS @ $10 \mathrm{ml} / \mathrm{kg}$ of seeds (Table 3) recorded highest B: C ratio of 4.95 and 4.50. The treatment Imidacloprid 17.8 SL $@ 0.3 \mathrm{ml} / \mathrm{Lt}$ of water, Dimethoate 30 EC @ $1.7 \mathrm{ml} / / \mathrm{Lt}$ of water, Neem oil (1500 ppm) @ $5 \mathrm{ml} / \mathrm{Lt}$ of water, seed treatment with Imidacloprid60 FS @ $5 \mathrm{ml} / \mathrm{Kg}$ of seeds and NSKE 5\% recorded 3.86, 3.58,3.20, 2.70, $2.21 \mathrm{~B}: \mathrm{C}$ ratio respectively. Untreated control recorded lowest $\mathrm{B}$ : $\mathrm{C}$ ratio of 1.74 .

\section{Effect on natural enemies}

The activity of the natural enemies was reduced in the treated plots compared to the untreated control as the population of the sucking pests was very low in treated plots.

\section{Phytotoxicity}

There was no Phytotoxicity symptoms were noticed on the crop after imposing the treatments. Further, it should be kept in the mind that, we need to sow the Imidacloprid 60 FS treated seeds when we have the 
optimum moisture/ enough moisture in the soil. As this is the first study by using seed treatment chemicals to manage the sucking pests of greengram, no reviews related to this topic is available in greengram. However, the other reviews are available by using Imidacloprid as a seed treatment to manage early stage sucking pests in cotton and bhendi; hence those reviews are used to compare the present results.

From the present study, the result revealed that Imidacloprid 60 FS @ 15 ml/ Kg of seeds and Imidacloprid 60 FS @ $10 \mathrm{ml} / \mathrm{kg}$ of seeds effectively reduced the sucking pest population in the greengram field. This result was almost similar to the findings of Mote et al., (1995) and Patil et al., (2003) who observed that Imidacloprid as seed treating chemical reduced sucking pest population below the economic threshold level up to 40 days after sowing in cotton and 61 days after germination (Dandale et al., 2001: Murugan et al., 2003) in cotton. Sreenivas and Nargund, 2006 reported that Imidacloprid @ $5 \mathrm{~g} / \mathrm{kg}$ of seeds as seed dressing chemical will protect the bhendi crop up to 50 days from sucking pests. Murugan and Kavitha (2009) reported that Imidacloprid recorded the least mean population of leafhoppers in cotton. The seed treatment by Imidacloprid recorded the lowest incidence of the sucking pests in blackgram Soundarajan and Chitra (2011). Imidacloprid (Gaucho 70 WS) effectively reduced population of aphids, whiteflies and thrips in cotton Hossain et al., 2013. Harish kumar et al., 2013 reported that Imidacloprid 600FS when applied as seed treatment was most effective in controlling the sucking pests up to four week of seed germination in soybean.

Imidacloprid60 FS @10 ml/kg seeds when applied as seed treatment when there is a sufficient soil moisture was most effective in controlling the sucking pests up to 40-45 days after germination with highest grain yield and cost effective.

\section{References}

Dandale, H. G., A. Y. Thakare, S. N. Tikar, N. G. V. Rao and S. A. Nimblakar. 2001. Effect of seed treatment on sucking pests of cotton and yield of seed cotton. Pestology. 25: 20-23.

Dhawan, A. K. and G. S. Simwat. 2002. Field evaluation of thiamethoxam for control of cotton jassid Amrasca biguttula (Ishida) on upland cotton. Pestology. 26: 15-19.

Harish kumar Netam, Rajeev gupta and Shivam soni. 2013. Bioefficacy of insecticides as seed treatment against early sucking pests of soybean crop. International Journal of Science and Research. 2 (1): 688-690.

Hossain, S. M.A., Baque, M. A., and Amin, M.R. 2013. Comparative effectiveness of seed treating and foliar insecticides against sucking pests of cotton and impact on their natural enemies. Bangladesh Journal of Agriculture research. 38 (1): 61-70.

Magalhaes, L. C., T. E. Hunt and B. D. Siegfried. 2009. Efficacy of neonicotinoid seed treatments to reduce soybean aphid populations under field and controlled conditions in Nebraska. J. Econ. Entomol. 102: 187-195.

Maienfisch, P., M. Angst, F. Brandl, W. Fischer, D. Hofer and H. Kayser. 2001. Chemistry and biology of thiamethoxam: a second generation neonicotinoid. Pest Manag. Sci. 57: 906-913.

Mote, U. N., R. V. Datkile and G. R. Loage. 1995. Efficacy of Imidacloprid as seed treatment against initial sucking pests of cotton. Pestology.19: 5-8.

Murugan, M. N. Sathiah, N. Dhandapani, R. J. Rabindra and S. Mohan. 2003. 
Laboratory assays on the role of Indian transgenic Bt cotton in the management of Helicoverpa armigera (Hubner) (Noctuidae: Lepidoptera). Indian J. Plant protection 31: 1-5.

Murugan, M., N. Sathiah, N. Dhandapani, R. J. Rabindra and S. Mohan. 2003. Laboratory assays on the role of Indian transgenic Btcotton in the management of Helicoverpa armigera (Hubner) (Noctuidae: Lepidoptera). Indian J. Plant Protection 31: 1-5.

Murugan, N. and Kavitha, A. 2009. Seed treatment with Pseudomonas fluorescens, plant products and synthetic insecticides against the leafhopper, Amrasca devastans (Distant) in cotton. Journal of Biopesticides, 2(1): 22-25.

Nault, B. A., A. G. Taylor, M. Urwiler,T. Rabaey and W. D. Hutchison. 2004. Neonicotiniod seed treatments for managing potato leafhopper infestations in snap bean. Crop Protection 23: 147154.

Patil, B. C., S. B. Patil, S. S. Vdikeri and B. M. Khadi. 2003. Effect of Imidacloprid seed treatment on growth, yield, seedling vigor and biophysical parameters in cotton (Gossypium spp) genotypes. In: Proc. World Cotton Res. Conf. 3, Cape Town, South Africa, 9-13 March 2003.

Patil, B. C., S. B. Patil, S. S. Vdikeri and B.M. Khadi. 2003. Effect of imidacloprid seed treatment on growth, yield, seedling vigor and biophysical parameters in cotton (Gossypium spp.) genotypes. In: Proc. World Cotton Res. Conf. 3, Cape Town, South Africa, 9-13 March 2003.

Rabindra, R.J., Ballali, C.R and Ramanujan B 2004. Biological options for insect pests and nematode management in pulses. Kalyani Publishers, New Delhi, India, 487.

Soundarajan, R. P. and Chitra, N. 2011. Effect of bioinoculants on sucking pests and pod borer complex in urdbean. Journal of Biopesticides, 4(1): 7-11

Sreenivas, A. G. and Nargund, V. B. 2006. Management of sucking insect pests of Bhendi through seed dressing chemicals. Karnataka Journal of Agricultural Sciences, 19(2): 307-311.

Taylor, A. G., C. J. Eckenrode and R. W. Straub. 2001. Seed coating technologies and treatments for onions: challenges and progress. Hort. Sci., 36: 199-205.

Udikeri, S. S., S. B. Patil, L. K. Naik, V.Rachappa, F. Nimbal and G. S. Guruprasad. 2007. Poncho 600 FS - a new seed dressing formulation for sucking pest management in cotton. Karnataka J. Agric. Sci., 20: 51-53.

Vadodaria, M. P., U. G. Patel, C. J. Patel, R. B. Patel and I. M. Maisuria. 2001. Thiamethoxam (Cruiser) 70 WS: a new seed dresser against sucking pests of cotton. Pestology, 25: 13-18.

Zhang, L., S. M. Greenberg, Y. Zhang and T. Liu. 2011. Effectiveness of thiamethoxam and imidacloprid seed treatments against Bemisia tabaci (Hemiptera: Aleyrodidae) on cotton. Pest Manag. Sci., 67:226-232.

\section{How to cite this article:}

Shobharani, M., Sidramappa and Sunilkumar, N.M. 2017. Evaluation of Different Doses of Imidacloprid 60 FS - a New Seed Dressing Chemical against Sucking Pests of Greengram. Int.J.Curr.Microbiol.App.Sci. 6(12): 3433-3441. doi: https://doi.org/10.20546/ijcmas.2017.612.399 